\title{
HSP90 Inhibitor Ganetespib (STA-9090) Inhibits Tumor Growth in c-Myc-Dependent Esophageal Squamous Cell Carcinoma
}

This article was published in the following Dove Press journal: OncoTargets and Therapy

\author{
Liuliu Guan ${ }^{1,2}$ \\ Qingqing Zou ${ }^{1,2}$ \\ Qian Liu ${ }^{2,3}$ \\ Yiguang Lin $\mathbb{D}^{3,4}$ \\ Size Chen ${ }^{1-3}$
}

'Department of Oncology, The First Affiliated Hospital of Guangdong Pharmaceutical University, Guangzhou, People's Republic of China; ${ }^{2}$ Guangdong Provincial Engineering Research Center for Esophageal Cancer Precise Therapy, Guangzhou, The First Affiliated Hospital of Guangdong Pharmaceutical University, People's Republic of China; ${ }^{3}$ Central Laboratory, The First Affiliated Hospital of Guangdong Pharmaceutical University, Guangzhou, People's Republic of China; ${ }^{4}$ School of Life Sciences, University of Technology Sydney, Sydney, NSW, Australia
Correspondence: Yiguang Lin School of Life Sciences, University of Technology Sydney, PO Box 123, Broadway, NSW 2007, Australia

Tel +6I 295142223

Fax +6I 295148206

Email yiguang.lin@uts.edu.au

Size Chen

Department of Oncology, The First Affiliated Hospital of Guangdong

Pharmaceutical University, 19 NonglinXia Road, Guangzhou 5I0080, People's

Republic of China

Tel +862061325337

Email chensize@gdpu.edu.au
Purpose: Currently, the paucity of classical effective pharmacological drugs to treat esophageal squamous cell carcinoma (ESCC) is a major problem. The c-Myc (MYC) protein is a promising target as it is overexpressed in ESCC. MYC is a sensitive client protein of the heat shock protein 90 (HSP90) and, therefore, targeting the HSP90-MYC axis by inhibition of HSP90 is a potential therapeutic strategy for ESCC. Here, we evaluated the clinical application value of the HSP90 inhibitor (Ganetespib, STA-9090) as an anti-cancer agent for MYC-positive ESCC.

Materials and Methods: We first analyzed ESCC tissue microarrays and clinical tissue samples to determine MYC expression. The relationship between MYC and HSP90 was analyzed by co-immunoprecipitation assays and immunofluorescence. In in vitro cell models, cell growth was analyzed using the CCK-8 kit, and MYC protein expression was analyzed by Western blot. The in vivo antitumor activity of STA-9090 was assessed in two xenograft animal models.

Results: We demonstrated that MYC-overexpressing ESCC cells were highly sensitive to STA-9090 treatment through suppressing ESCC cell proliferation, cell cycle progression and survival. Moreover, STA-9090 treatment decreased MYC expression, reducing the half-life of the MYC protein. We further established two xenograft mouse models using ESCC cells and clinical ESCC samples to validate the effectiveness of STA-9090 in vivo. In both xenograft models, STA-9090 substantially inhibited the growth of MYC-positive ESCC tumors in vivo. In contrast, STA-9090 treatment demonstrated no beneficial effects in mice with low-MYC expressing ESCC tumors.

Conclusion: In conclusion, our data support that the HSP90 inhibitor, STA-9090, suppresses the expression of the MYC protein and interferes with HSP90-MYC protein-protein interaction. This, in turn, leads to inhibition of ESCC cell proliferation and promotion of apoptosis in ESCC cells in vitro and reduction of ESCC tumors in vivo. We propose, based on our findings, that STA-9090 is a potential novel therapeutic target for MYC-positive ESCC.

Keywords: esophageal squamous cell cancer, c-Myc, HSP90 inhibition, Ganetespib, STA-9090, patient-derived xenograft model

\section{Introduction}

Esophagus Cancer (EC) is a common malignant tumor ranked seventh and sixth in terms of prevalence and mortality rate respectively. ${ }^{1}$ The major classifications for EC pathology are esophagus adenocarcinoma (EAC) and esophagus squamous cell carcinoma (ESCC), with ESCC accounting for approximately $90 \%$ of all esophageal cancer cases worldwide. Recent epidemiological data show that approximately three quarters 
of new cases develop in countries with low to medium levels of socioeconomic development, and nearly half (49\%) of all new cases occur in China. ${ }^{2}$ Many patients with ESCC are diagnosed at an advanced stage, and the mainstay treatments for ESCC such as surgery, chemotherapy, and radiotherapy have limited efficacy. ${ }^{3}$ The prognosis of EC is poor with a five-year survival rate of less than $20 \%$. ${ }^{4}$ Although there are a few targeted drugs, such as monoclonal antibodies and tyrosine kinase inhibitors, 3,5 that play important roles in treating advanced ESCC tumors, their effectiveness are still lacking. ${ }^{6}$ Therefore, there is a need for in-depth studies of the molecular mechanism of ESCC aiming at the discovery of new therapeutic targets for better patient outcome.

C-Myc (MYC) is a member of a family of protooncogenes, which are transcription factors that regulate target gene transcription and induce malignant cell growth and proliferation. $^{7-9}$ Members of the MYC family promote DNA synthesis and are important mediators in the transition from the $\mathrm{G} 1$ to $\mathrm{S}$ phase in the cell cycle. It is estimated that MYC regulates $15 \%$ of gene expression in humans and promotes the expression of genes involved in tumor proliferation. ${ }^{10}$ The MYC protein has been reported to be commonly over-expressed in ESCC tumors with a positive expression rate of $61.05 \% .^{11}$ Recently, since starting this project, targeted MYC therapy using the bromodomain inhibitor, JQ1, has shown to be effective in suppressing ESCC tumors in pre-clinical models. ${ }^{12}$

The heat shock protein 90 (HSP90) is a key regulator molecule that maintains protein homeostasis and cell survival. ${ }^{13}$ HSP90 is highly expressed in several cancers, including ESCC. ${ }^{14-16}$ Many client proteins of HSP90 are involved in cell cycle progression and cell survival. Inhibition of HSP90 leads to the degradation of client proteins and induces cell cycle arrest or apoptosis. ${ }^{17} \mathrm{MYC}$ is a crucial client protein of HSP90 and the HSP90-MYC complex is important in cell cycle progression. ${ }^{18}$ The drug Ganetespib (STA-9090) is a second-generation HSP90 inhibitor that presents potent cytotoxicity in a range of solid and hematological tumors and demonstrates antitumor activity with promising safety profiles in vivo in a variety of cancers. ${ }^{19}$ At present, many Phase I-III clinical trials of STA-9090 for human cancer treatment are ongoing, including breast and lung cancers. ${ }^{20-24}$ As the HSP90-MYC has been shown to be critical in cancer cell proliferation and given MYC is highly expressed in aggressive ESCC resistant tumors, in this study we investigated the clinical significance and antitumor effects of STA-9090 on MYC-positive ESCC.
In this study, we used an alternative strategy to inhibit MYC function through blocking a critical protein-protein, HSP90-MYC, interaction to alter the stability and function of MYC and evaluate its potential as a viable treatment option for ESCC patients with high MYC expression. We first analyzed MYC expression in ESCC tissue microarrays and cancer tissue samples from patients with ESCC and then defined the interaction between MYC and HSP90 in ESCC through in vitro and in vivo experiments. Utilizing ESCC cell lines and two animal xenograft models, we investigated the role of the HSP90 inhibitor, STA-9090, in ESCC carcinogenesis, aiming to uncover a potential therapeutic target for ESCC through blocking the HSP90-MYC axis.

\section{Materials and Methods}

\section{Reagents}

Phosphate buffered saline (PBS), Dulbecco minimal essential medium (DMEM), Roswell Park Memorial Institute (RPMI)1640 and fetal bovine serum (FBS) were purchased from Gibco (Thermo, UT, USA). Short interfering RNAs (siRNAs) targeting MYC were purchased from GenePharma (Shanghai, China). Lipofectamine 2000 and TRIzol were purchased from Invitrogen (Carlsbad, CA, USA). PrimeScript ${ }^{\mathrm{TM}}$ RT reagent KIT and TB Green ${ }^{\mathrm{TM}}$ Premix Ex Taq ${ }^{\mathrm{TM}}$ II were from Takara (Otsu, Shiga, Japan). The CCK-8 proliferation kit was purchased from Dojindo Laboratories (Kumamoto, Japan). RIPA (Radio Immunoprecipitation Assay) Lysis Buffer, Bicinchoninic acid (BCA) protein assay kit, and Propidium Iodide (PI) kit were supplied by Beyotime Biotechnology (Shanghai, China).

\section{Pharmacologic Agents}

Cycloheximide (CHX) and STA-9090 were obtained from Selleck Chemicals. Stock solutions of CHX (10 $\mathrm{mmol} / \mathrm{L})$ and STA-9090 $(10 \mathrm{mmol} / \mathrm{L})$ were stored at $-20^{\circ} \mathrm{C}$. For in vivo studies, STA-9090 (5mg/mL) was diluted with 10\% DMSO, 18\% Cremophor RH 40 (BASF), 3.6\% dextrose (Sigma, St. Louis, MO, USA) and 68.4\% water. STA-9090 treatments of mice with STA-9090 were carried out by intraperitoneal injection at the dose of $25 \mathrm{mg} / \mathrm{kg} / \mathrm{d}$ every 3 days, totaling 7 injections.

\section{Tissue Specimens}

Twelve ESCC tissue samples (9/male, 3/female) were collected from the First Affiliated Hospital of Guangdong Pharmaceutical University between 2016 Dec and 2019 
Oct. ESCC tumor staging was performed according to the AJCC Eighth Edition Cancer Staging Manual. (Details in Supplementary Table 1). The study was approved by the Human Ethic Committee of the First Affiliated Hospital of Guangdong Pharmaceutical University, and all tissue sample collections were performed with written informed consent from the patients. The study was conducted in accordance with the Declaration of Helsinki.

\section{Cell Culture}

ESCC cell lines (KYSE150, TE-1, TE-13, Eca-109, EC-1) were acquired from SGST (Shanghai, China). Noncarcinoma human esophageal epithelial cells (HEECs) were purchased from BeNa Culture Collection (Beijing, China). All ESCC cells were cultured in DMEM containing 10\% FBS. HEEC was cultured in RMPI 1640 with $10 \%$ FBS. All cells were incubated in a $5 \% \mathrm{CO}_{2}$ incubator at $37^{\circ} \mathrm{C}$.

\section{Cell Proliferation}

The CCK-8 kit analysis was used to investigate cell proliferation. Briefly, the cells were seeded into 96-well plates and incubated for $24 \mathrm{~h}$, then treated with control or Ganetespib (STA-9090) for 72 h. Finally, $10 \mu \mathrm{L}$ CCK-8 solution was added to each well and incubated for $2 \mathrm{~h}$. Absorbance $(450 \mathrm{~nm})$ was measured. The $\mathrm{IC}_{50}$ results were calculated with PRISM6 software (GraphPad, La Jolla, USA).

\section{Immunofluorescence}

For immunofluorescence analyzes, cells were plated on glass slides in 6-well plates. After $24 \mathrm{~h}$, different concentrations of Ganetespib (STA-9090) or control (vehicle) were added to cells for $72 \mathrm{~h}$. Cells were fixed with $4 \%$ paraformaldehyde. The fixed cells were incubated with $3 \%$ BSA for $30 \mathrm{~min}$ and then overnight with the MYC antibody (1:50) at $4^{\circ} \mathrm{C}$. After washing (x3) with PBS, the cells were incubated with goat-anti-rabbit antibody (1:50) for $50 \mathrm{~min}$ and mounted using fluorescent mounting medium (Beyotime, China). Cells were examined using a Nikon upright fluorescence microscope (NIKON, Japan) and at least 1000 cells from 10 to 15 viewing fields per group were used to calculate percentages of cells.

\section{Quantitative Real Time (qRT)-PCR}

For qRT-PCR, total RNA from cell samples was prepared using TRIzol. Reverse transcription of RNA and qRT-PCR were performed using an Analytikjena qTower3g Real-
Time PCR System (Analytikjena, Jena, German). The primer sequences are listed in Supplementary Table 2.

\section{Short Interfering RNA (siRNA) Transfection}

ESCC cells were seeded into 6-well plates and cultured overnight. Using Lipofectamine 2000, ESCC cells were then transfected with MYC-siRNA or ctrl-siRNA according to the manufacturer's instructions.

\section{Western Blot}

ESCC cells were plated into 6-well plates at a density of $2.5 \times 10^{5} /$ well. After 24 hrs STA-9090 or vehicle was added into designated wells for $72 \mathrm{~h}$. The cells were lysed with RIPA Lysis Buffer and protein concentrations were measured with a BCA assay kit. The protein lysates were electrophoresed and transferred to PVDF membranes (GE Healthcare, Buckinghamshire, UK) and incubated overnight with the primary antibodies at $4^{\circ} \mathrm{C}$. After washing with PBS the membranes were incubated with the appropriate special secondary antibodies at room temperature for $1 \mathrm{~h}$ for signal detection. Antibodies used: HSP90 (1:1000) antibody and $\beta$ actin $(1: 10,000)$ antibody (Affinity Biosciences, OH, USA), BAX (1:500), BCL-2 (1:500), MYC (1:500) antibody (ABclonal Biotech Co, Woburn, MA, USA), Anti-mouse HRP $(1: 10,000)$ (ABclonal Biotech Co, Woburn, MA, USA), Anti-rabbit HRP (1:10,000) (ABclonal Biotech Co, Woburn, MA, USA).

\section{Cell Cycle Analysis}

Following treatment with control or STA-9090 for $24 \mathrm{~h}$, KYSE-150, Eca-109 and TE-1 cells were collected and fixed in $70 \%$ ethanol at $4{ }^{\circ} \mathrm{C}$ overnight. The cells were then stained with propidium iodide $(\mathrm{PI})$ at $37^{\circ} \mathrm{C}$ for $30 \mathrm{~min}$. Data were acquired by FACS Vantage flow cytometer (BD Biosciences, USA). The proportions of cells at each cell cycle stage were determined using ModFit software (Verity Software House, USA).

\section{Apoptosis Analyses}

To study the apoptosis of different cell types, $5 \times 10^{5}$ cells per well were seeded into 6-well plates and cultured with control or STA-9090 for $24 \mathrm{~h}$, then the cells were harvested and stained with Annexin V-FITC/PI, and analyzed using FlowJo software (FlowJo LLC, USA). 


\section{Co-Immunoprecipitation Assay (Co-IP)}

The cells were lysed using lysing buffer $(150 \mathrm{mM} \mathrm{NaCl}$, 50mM Tris-HCl, $\mathrm{pH} 7.4,1 \% \mathrm{NP}-40$, sodium pyrophosphate, $\beta$-glycerophosphate, sodium orthovanadate, sodium fluoride, leupeptin and fresh protease inhibitor mixture). The lysates were incubated with antibodies/protein $\mathrm{A}+\mathrm{G}$-conjugated agarose beads (Beyotime Biotechnology, Shanghai, China). Rabbit anti-mouse IgG antibody (Beyotime Biotechnology, Shanghai, China) was used as a control. The precipitates were washed with lysis buffer ( $\mathrm{x} 5$ ), then resuspended in $2 \mathrm{x}$ loading buffer followed by Western blot.

\section{H\&E Staining and Immunohistochemistry}

Tumor tissues were cut as $4 \mu \mathrm{m}$ sections, then stained with a hematoxylin and eosin (H\&E) kit. For immunohistochemical experiments, the sections were incubated with Ki-67 (1:50) antibody, cleaved-Caspase 3(1:50) antibody and MYC (1:50) antibody at $4{ }^{\circ} \mathrm{C}$ respectively, and counterstained with hematoxylin. Positive stained cells were counted by two independent experienced researchers. Expression status was divided into three groups according to the percentage of the positive cells (Negative: $<20 \%$ cells positive; Weak: $20-40 \%$ cells positive; and Intermediately to strong: $>40 \%$ cells positive).

\section{ESCC Tissue Microarrays (TMA)}

ESCC tissue microarrays (HEso-S180Su-08) were purchased from Shanghai Outdo Biotech Co. The primary antibodies against HSP90 (1:200) or MYC (1:50) were diluted and then incubated at $4{ }^{\circ} \mathrm{C}$ overnight. The staining analysis of HSP90 and MYC was divided into three groups (Negative: $<20 \%$ cell positive; Weak: $20-40 \%$ cell positive; Intermediately to strong: $>40 \%$ cell positive). Samples were excluded in the event of TMA shedding, unclear clinical diagnosis and inappropriate technical quality.

\section{Xenograft Experiments}

ESCC cell-derived xenograft (CDX) mouse models were induced by subcutaneous injection of $1 \times 10^{6}$ cells of KYSE150 or TE-1 cells with 100 ul of (1:1) PBS/Matrigel (BD Biosciences, Franklin, NJ, USA), into the dorsal side of nude mouse. Drug therapy was commenced when the tumor reached approximately $5 \mathrm{~mm}$ in diameter ( $\mathrm{n}=5$ per group). The experiments were approved by the Animal Care and Use Committee of the First Affiliated Hospital of Guangdong Pharmaceutical University, and animal welfares were closely monitored in accordance with the Guidelines for the Care and Use of Experimental Animals of Guangdong Province.

\section{ESCC PDX Models}

The ESCC patient-derived xenograft (PDX) models were established using the methods below. The patient ESCC samples for grafting were received from the First Affiliated Hospital of Guangdong Pharmaceutical University. The ESCC tumor tissues excised from the patients were cut up and transplanted into the back of the 5 to 6-week-old female $\mathrm{B}-N D G^{\circledR}(\mathrm{NSG})$ mice (Biocytogen, Beijing, China. When tumor xenografts were formed, we defined these tumors as the first generation (P1). Once the P1 generation tumors reached $1200 \mathrm{~mm}^{3}$ in size mice were sacrificed, and the tumors were removed for the next generation of transplantation (P2). Using the same tumor transplantation method, the third generation (P3) of ESCC PDX mice models were developed for in vivo experiments ( $\mathrm{n}=5$ per group).

\section{Calculation of Tumor Growth}

Tumor size was assessed (every day) using a vernier caliper. The formula for calculating tumor volume was $\mathrm{V}=\mathrm{d}^{2}$ $\times \mathrm{D} / 2$ ( $\mathrm{d}=$ the shortest diameter, $\mathrm{D}=$ the longest diameter). The results were averaged by three measurements.

\section{Statistical Analysis}

Data were analyzed using SPSS software version 22.0 and PRISM6 Software. For TMA, the relationship between MYC expression, HSP90 expression, and the clinical pathologic parameters was examined using Pearson $\chi^{2}$ test. For the clinical samples, the differences in MYC expression level between ESCC tissues and matching adjacent nontumorous tissues were assessed by the paired-sample $t$-test. In vitro studies, for all experiments involving statistical analysis, the Student's $t$-test was used for two groups while one-way ANOVA analysis was used for multiple group comparisons. Unpaired Student's $t$-test was used in all in vivo studies. Statistical significance was indicated by an asterisk ( $* \mathrm{P}<0.05, * * \mathrm{P}<0.01, * * * \mathrm{P}<0.001)$.

\section{Results}

\section{MYC Was Found to Be Overexpressed}

\section{and Positively Associated with HSP90} Expression in ESCC Tissues

To determine whether ESCC could be treated by targeting MYC with the inhibition of HSP90, we analyzed the expression of MYC and HSP90 using a TMA of 107 ESCC samples. Immunohistochemical staining showed that MYC-positive expression rate was present in over half of the samples (Figure 1A and B). The intermediate/strong levels of HSP90 


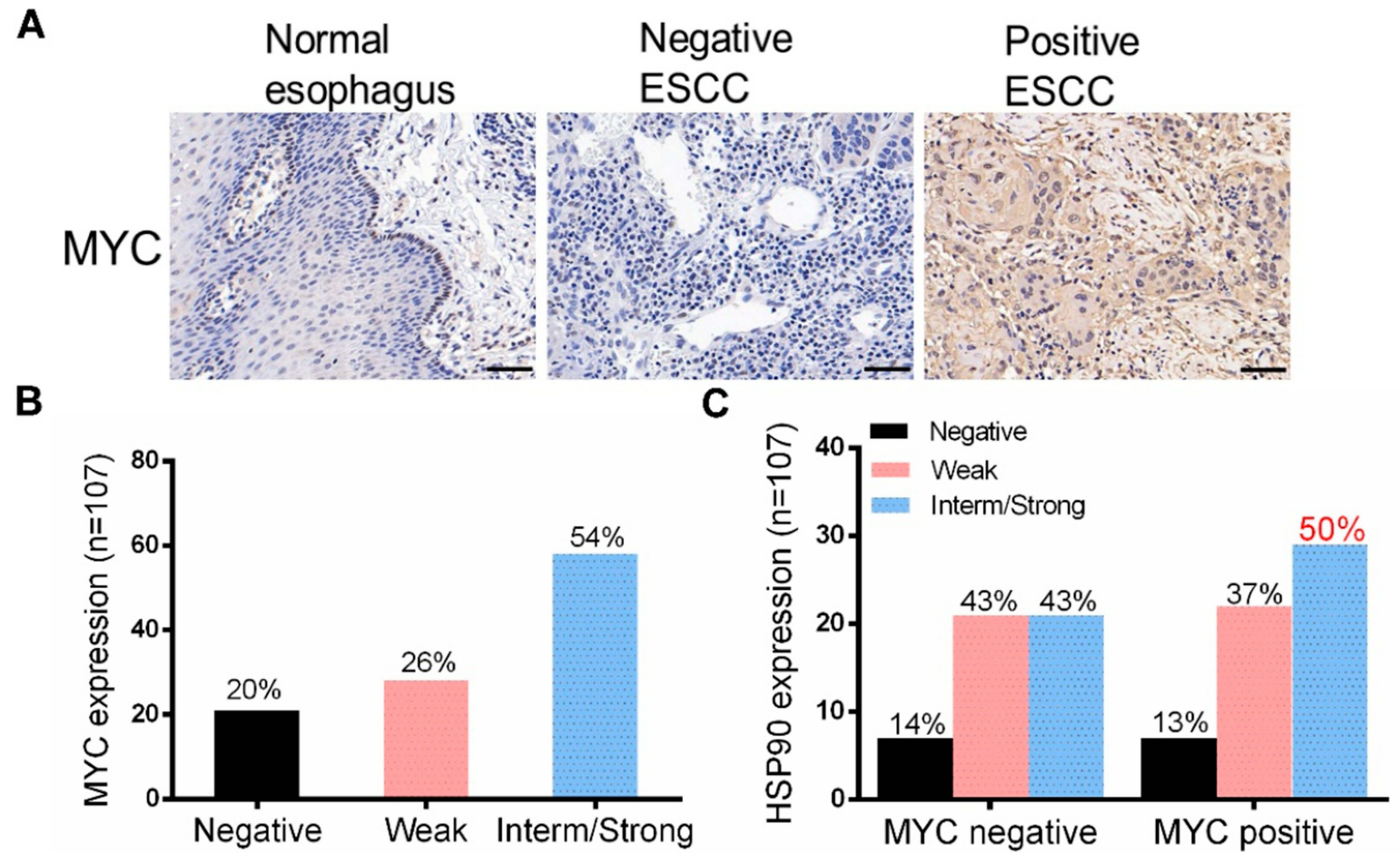

D
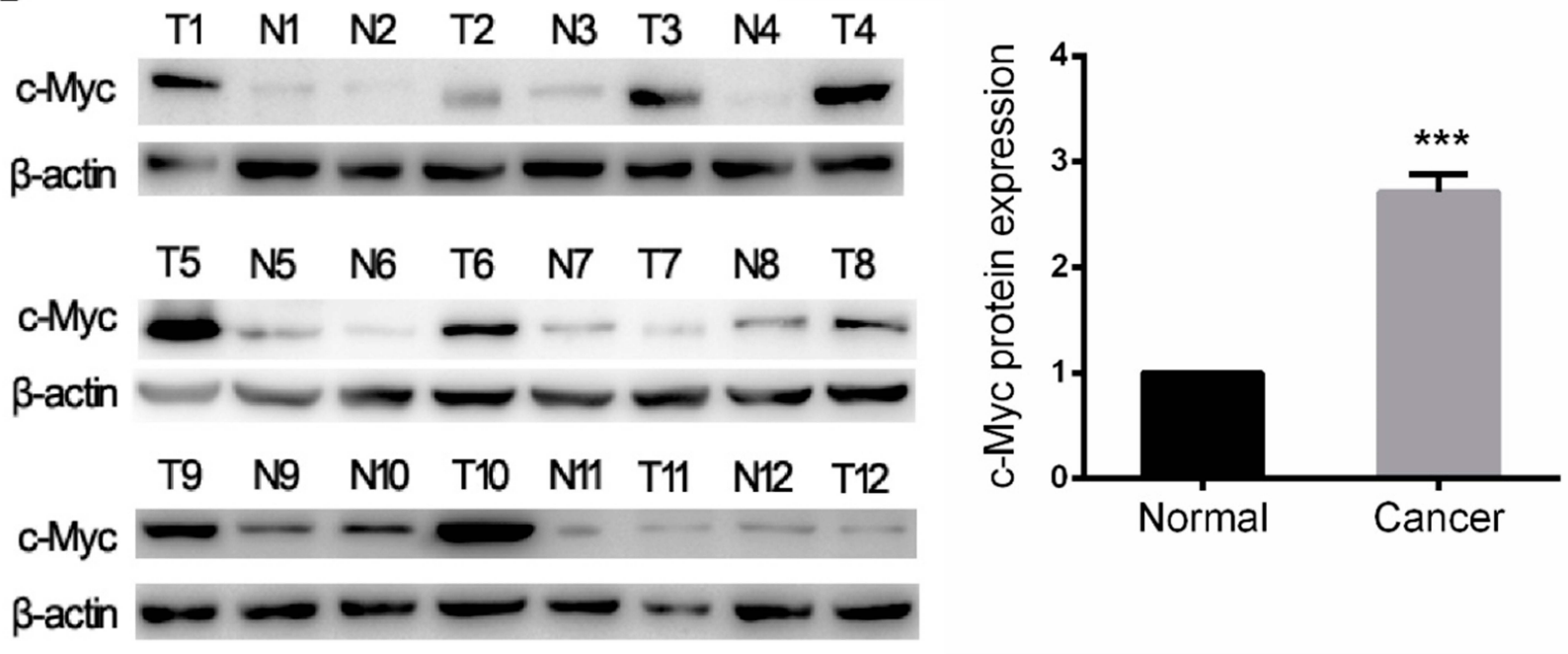

E

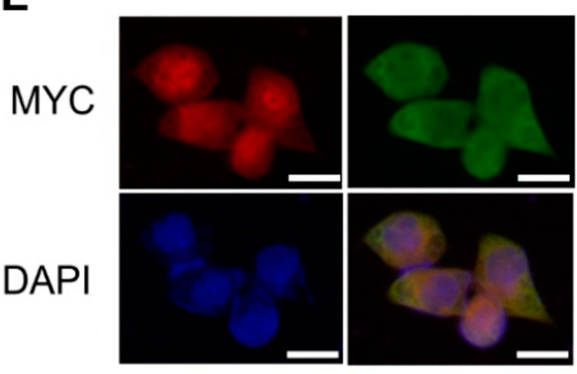

$\mathbf{F}$

Figure I MYC was over-expressed and interacted with HSP90 in ESCC. (A and B) MYC' nuclear expression in ESCC. Images from TMA immunostained for MYC. Scale bars represent $50 \mu \mathrm{m}$. (C) MYC expression against HSP90 expression status showing $50 \%$ of double-positive samples. (D) Western blotting examined the expression of MYC in ESCC tumor tissues and adjacent normal tissues $(n=12)$. (E) Localization of HSP90 and MYC in Eca-109 cells by immunofluorescence. Scale bars represent $25 \mu$ m. (F) Interaction of endogenous HSP90 with MYC was detected by co-IP assays in Eca-109 cells. ***P $<0.001$. 
was approximately $\geq 47 \%$ cases of all tumors (Supplementary Figure 1A and B). HSP90 was highly expressed in $50 \%$ of the ESCC with MYC-high status (Figure 1C). Therefore, high expression of MYC and HSP90 protein were found to be common features in ESCC. We then detected the MYC protein expression in twelve tumor tissues by Western blot and discovered that MYC was highly expressed in tumors compared to adjacent normal tissues in $58.3 \%(7 / 12)$ of the patients (Figure 1D).

\section{MYC Interacts with HSP90 in ESCC Cells}

In order to determine whether HSP90 physically interacts with MYC in ESCC cells, first we used immunofluorescence imaging to validate that HSP90 and MYC were co-localized in the nucleus of ESCC cells (Figure 1E). Co-immunoprecipitation (co-IP) assays showed that the MYC could interact with HSP90 in Eca-109 cells (Figure 1F).

To examine the specific effects of STA-9090 on MYC protein stability in ESCC cell, we treated Eca-109 with CHX $(100 \mu \mathrm{g} / \mathrm{mL})$, a protein synthesis inhibitor, alone or in combination with STA-9090 $(1 \mu \mathrm{M})$ and collected cells at specified time points. As shown in Supplementary Figure 2, MYC protein expression was significantly decreased after 4 hrs with CHX + STA-9090 treatment, nevertheless, CHX treatment alone decreased after $12 \mathrm{hrs}$ in Eca-109 cells.

\section{MYC-Overexpressing ESCC Cells Were More Sensitive to STA-9090}

Expression of MYC proteins was assessed in five human ESCC cell lines and overexpression was found in two cell lines, KYSE-150 and Eca-109 (Figure 2A and B). Following STA-9090 treatment for $72 \mathrm{hrs,} \mathrm{we} \mathrm{found} \mathrm{that} \mathrm{STA-9090}$ inhibited the proliferation of ESCC cell lines in a dosedependent manner and MYC overexpressing ESCC cells were highly sensitive to STA-9090 treatment. The $\mathrm{IC}_{50}$ value of KYSE-150 and Eca-109 is $29.32 \mathrm{nM}$ and 69.44 $\mathrm{nM}$, respectively (Figure 2C and Supplementary Table 3).

In MYC overexpressing cell lines, KYSE-150 and Eca109, STA-9090 treatment significantly generated G1 phase arrest, with a decrease in both $\mathrm{S}$ and G2/M phase population, when compared to control treatment (Figure 2D and Supplementary Figure 3). However, in the STA-9090 insensitive ESCC cell line, TE-1, STA-9090 did not induce any G1 changes. Similarly, we observed an increase in cell cycle-dependent kinase inhibitors, represented by $\mathrm{p} 15$ (encoded by CDKN2B) and p21 (encoded by CDKN1A) which are repressed by MYC at the transcriptional level and the key regulators of G1 checkpoint. ${ }^{25}$ We found that STA-9090 treatment significantly induced $\mathrm{p} 21$ and $\mathrm{p} 15$ mRNA expression in STA-9090 sensitive cells KYSE150 and Eca-109 (Figure 2E), while TE-1 cells exhibited decreased expression levels. Our results showed that STA9090 inhibited tumor cell proliferation, ultimately causing cell cycle arrest in ESCC cells.

\section{STA-9090 Inhibited ESCC Proliferation and Promoted Apoptosis by Down-Regulating MYC Expression}

STA-9090 treatment decreased MYC protein expression in a dose-dependent manner in all cell lines when compared to the control, as shown by the Western blotting results Figure $3 \mathrm{~A}$ and B. Decrease in MYC expression in STA9090 treated cells was also validated by immunofluorescence imaging (Figure 3C). Apoptosis of MYC-overexpressed ESCC cells was significantly increased following STA9090 treatment (Figure 3D). STA-9090 treatment also led to concurrent increase and decrease in pro- and antiapoptosis proteins, BAX and BCL-2, respectively (Figure 3E and F). To test whether MYC promotes proliferation of the ESCC cell lines, we used MYC siRNAs to perform specific cell cycle inhibition in three cell lines. MYC expression was successfully silenced in all 3 cell lines TE-1 cell, KYSE150 and Eca-109 cells (Figure 4A and B) as confirmed by Western blot analysis. MYC silencing remarkably inhibited cell proliferation (Figure 4C). Cell apoptosis was significantly increased after transfecting MYC-siRNA (Figure 4D). These results demonstrated that the inhibition of HSP90 activity using STA-9090 decreased MYC expression and caused downregulation of cell proliferation and increased cell apoptosis in ESCCs.

\section{STA-9090 Inhibited ESCC Tumor Growth in CDXs and Downregulated MYC Expression}

To evaluate the in vivo effect of inhibition of HSP90 by STA-9090, we established CDX mouse models. We tested the treatment outcome of STA- 9090 by intraperitoneal injection of vehicle control or STA-9090 $(25 \mathrm{mg} / \mathrm{kg})$ every three days for 21 days, a relevant dose in human cancer therapy. ${ }^{26}$ We found that STA-9090 treatment significantly inhibited KYSE-150 tumor growth and retarded TE-1 tumor growth (Figure 5A, B and E) compared to vehicle-treated control mice. In mice with KYSE-150 
A

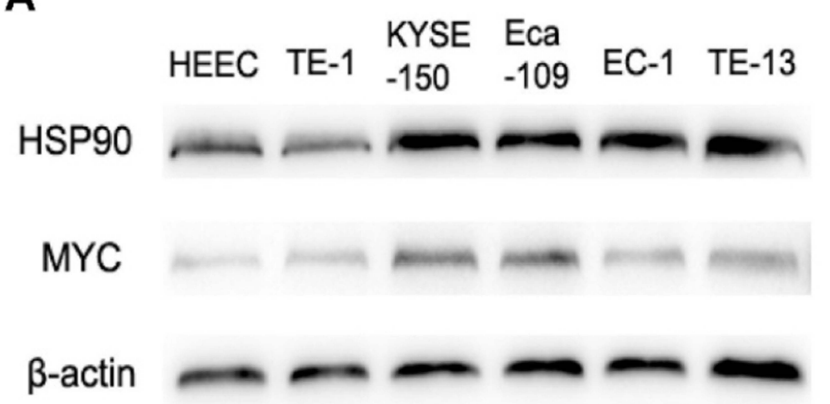

B

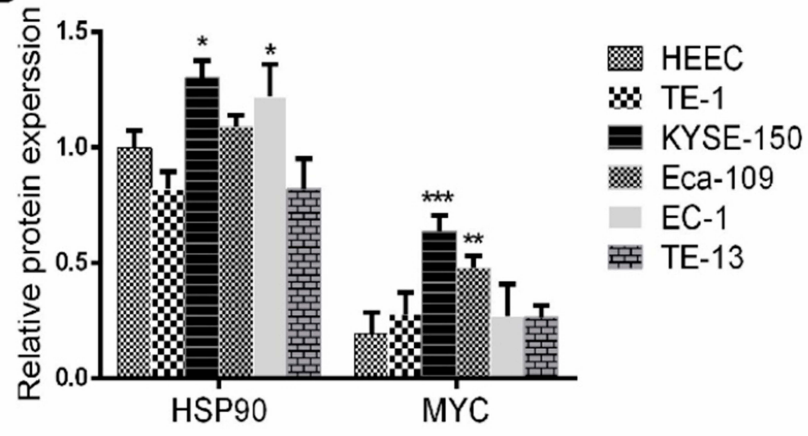

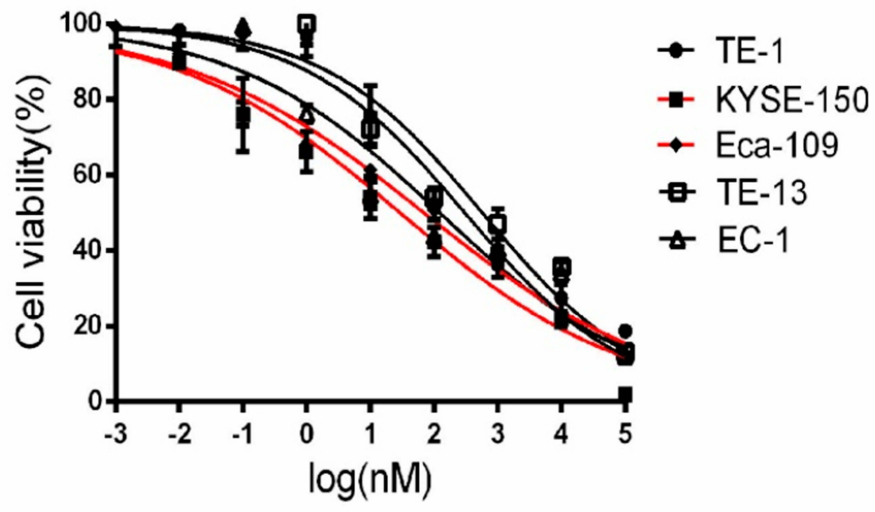

D

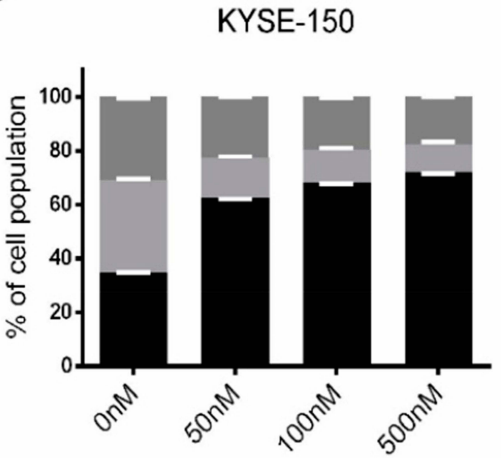

Eca-109

TE-1
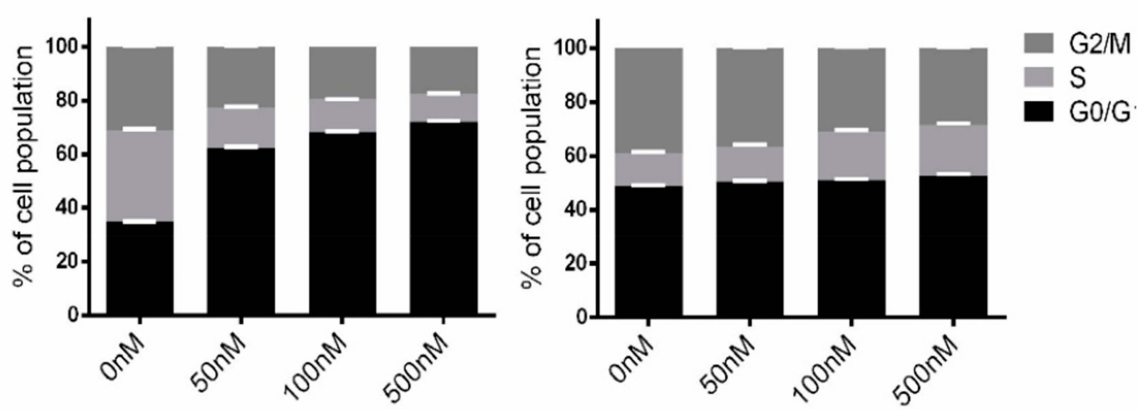

E
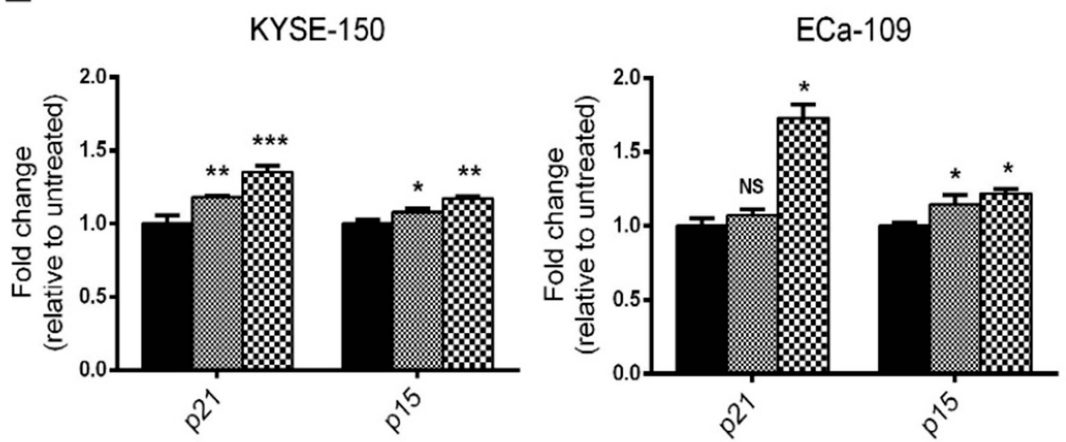

TE-1

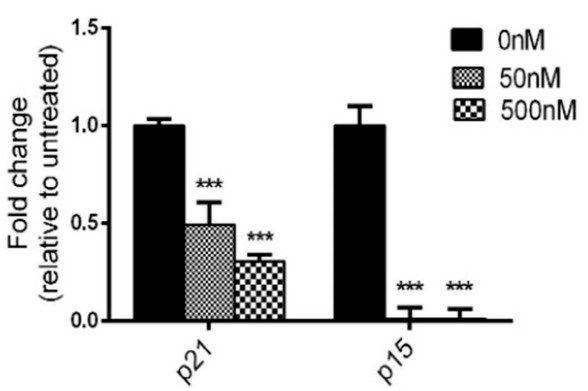

Figure 2 MYC-overexpressing ESCC cell lines were more sensitive to STA-9090. (A and B) Western blotting were used to analyze levels of MYC and HSP90 in the indicated ESCC cells and HEEC. (C) Growth inhibition curves of STA-9090 in five ESCC cell lines. Red curves represent are sensitive to STA-9090. (D) Cell cycle distributions of KYSE-150, Eca- 109 and TE-I cells treated with different concentrations of STA-9090. (E) qRT-PCR revealed STA-9090 induces P2I and pI5 mRNA expression in KYSE-150, Eca-109 and TE-I cells were treated with STA-9090 for 24 hrs. $* \mathrm{P}<0.05$, **P $<0.01$, *** $\mathrm{P}<0.00 \mathrm{I}$. 
A
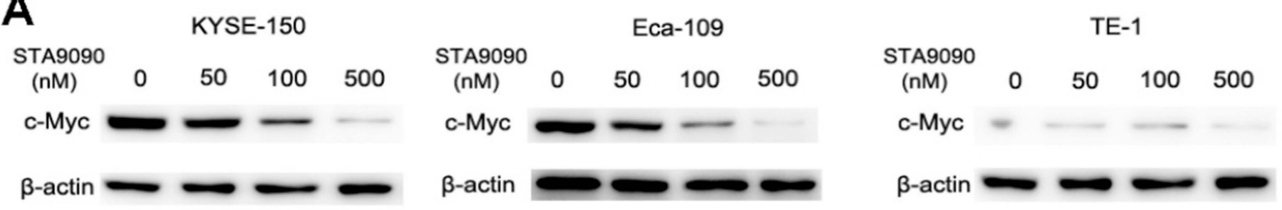

B
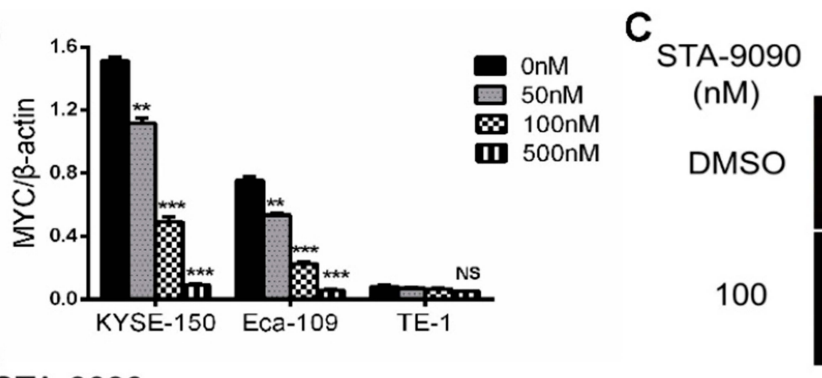

MYC

DAPI

Merge

DMSO

100
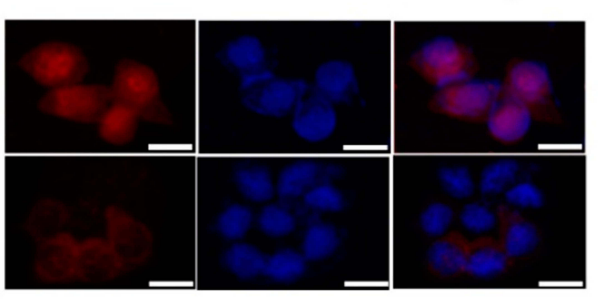

STA-9090

(nM)

PI

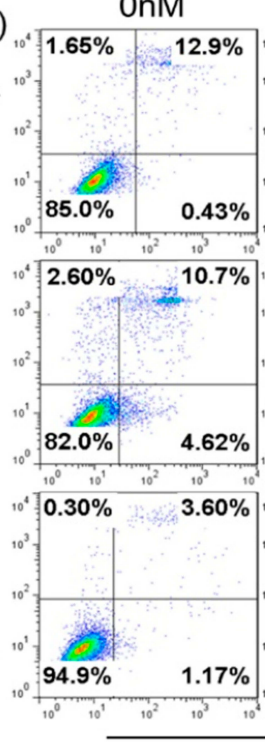

\section{$50 \mathrm{nM}$}

100nM

500nM

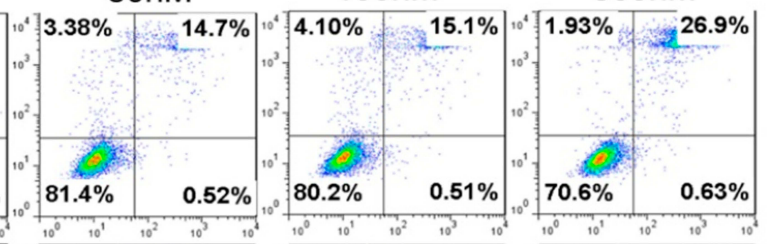

Eca-109

KYSE-150

E

KYSE-150

Annexin V FITC

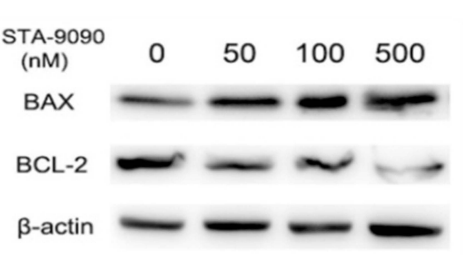

F KYSE-150
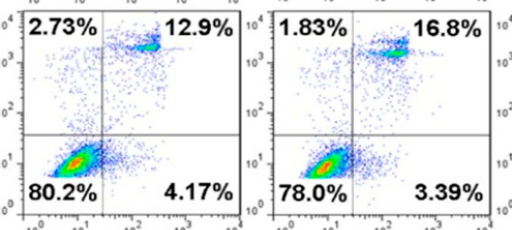

\begin{tabular}{|l|l|}
$21.2 \% \quad 19.7 \%$ \\
\hline
\end{tabular}

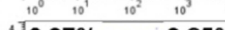

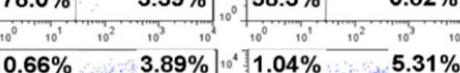
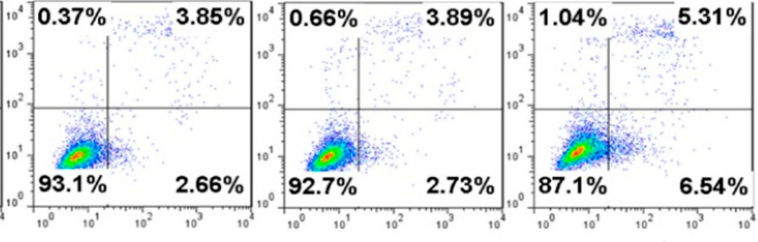

TE-1
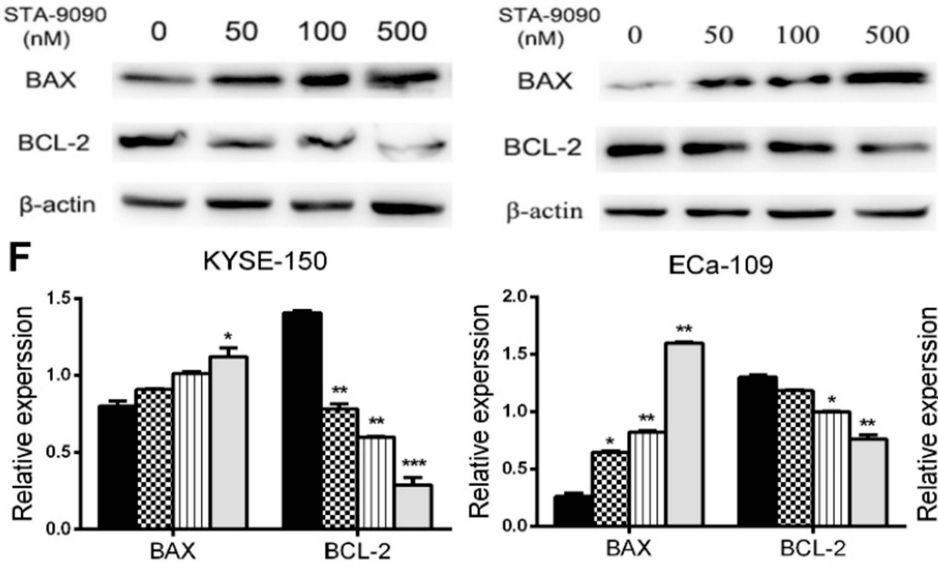

ECa-109

TE-1

TE-1

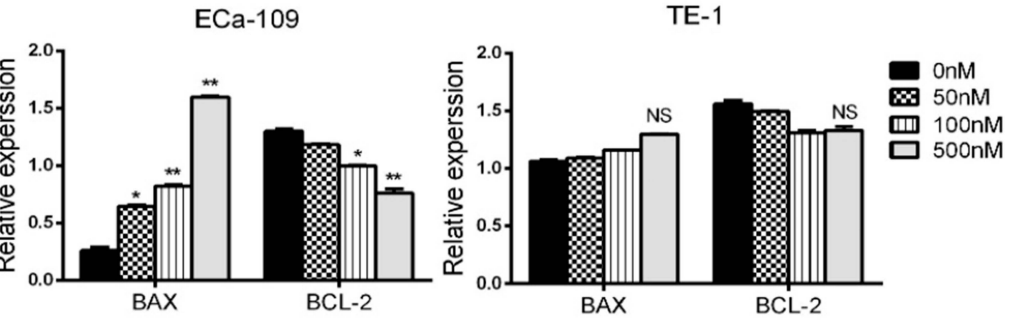

Figure 3 STA-9090 inhibited MYC expression and promoted apoptosis of ESCC cells. (A, B) KYSE-150, Eca-109 and TE-I cells protein expression of MYC was detected by Western blotting. (C) MYC was detected with STA-9090-treated compared to control group in Eca-109 cell by immunofluorescence. Scale bars represent $25 \mu \mathrm{m}$. (D) Flow cytometry analyzed KYSE-150, Eca-109, TE-I cell apoptosis levels with Annexin V/PI staining. $X$ axis: Annexin V staining; $Y$ axis: PI staining. (E, F) Proteins expression of BCL-2 and BAX were detected by Western blotting in KYSE-150, Eca-109, TE-I cells with STA-9090 treatment. $* \mathrm{P}<0.05$, $* * \mathrm{P}<0.01$, $* * * \mathrm{P}<0.001$.

Abbreviation: NS, no significance. 
A

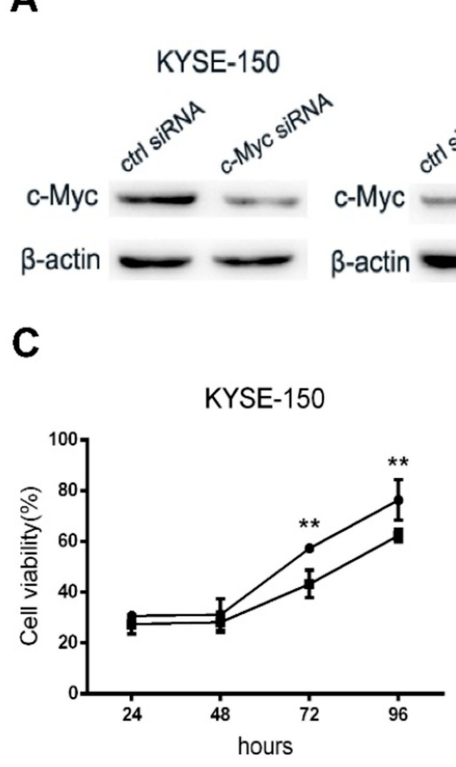

B

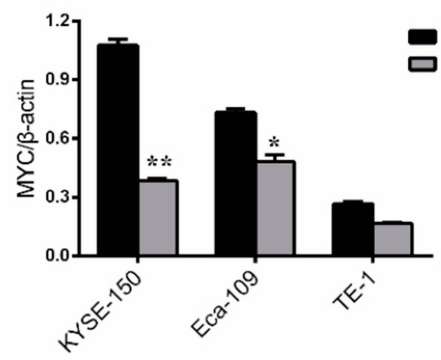

D

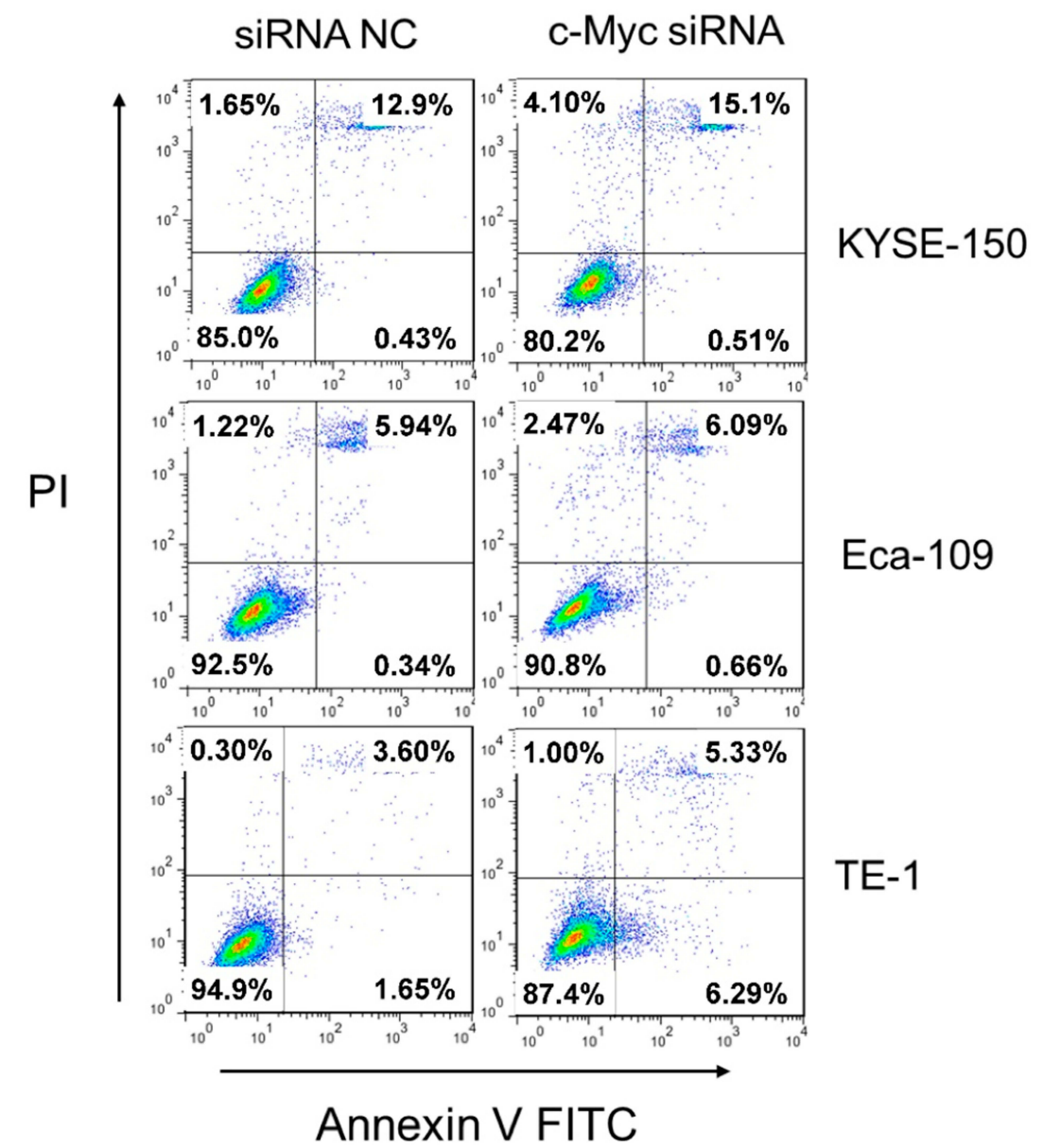

Figure 4 Silencing of MYC inhibited tumor cell growth. (A, B) KYSE-150, Eca-109 and TE-I cells were treated with MYC siRNA and ctrl siRNA for 48 hrs, and then detecting the expression levels of MYC. (C) Growth of MYC siRNA or ctrl siRNA-transfected KYSE-150, Eca-109 and TE-I cells over the course of 96 hrs. (D) Apoptosis measured by Annexin V/PI double staining in three cells transfected with MYC and ctrl siRNA for 48 hrs. $* \mathrm{P}<0.05, * * \mathrm{P}<0.01$. 
A

KYSE-150

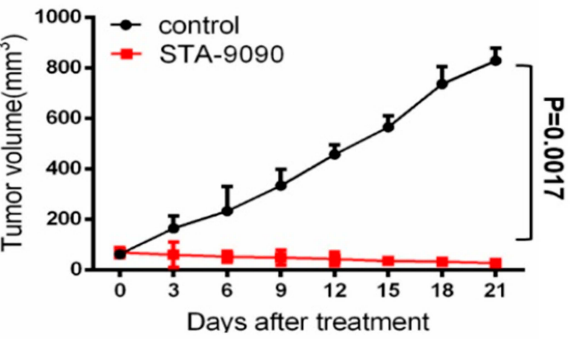

KYSE-150

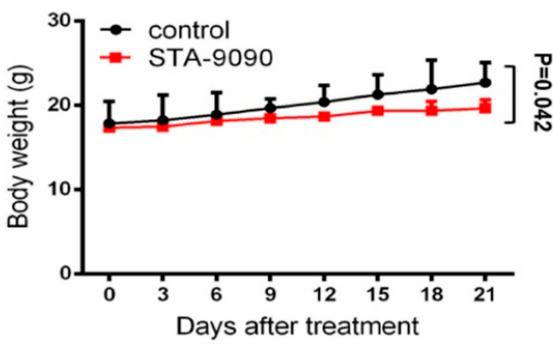

E

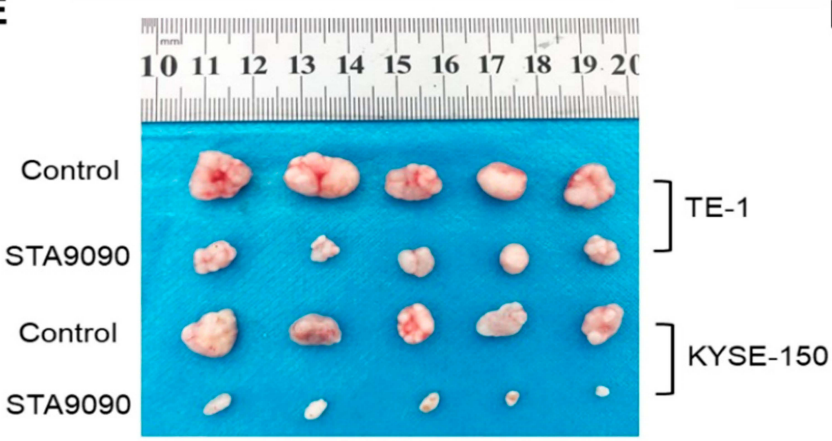

G

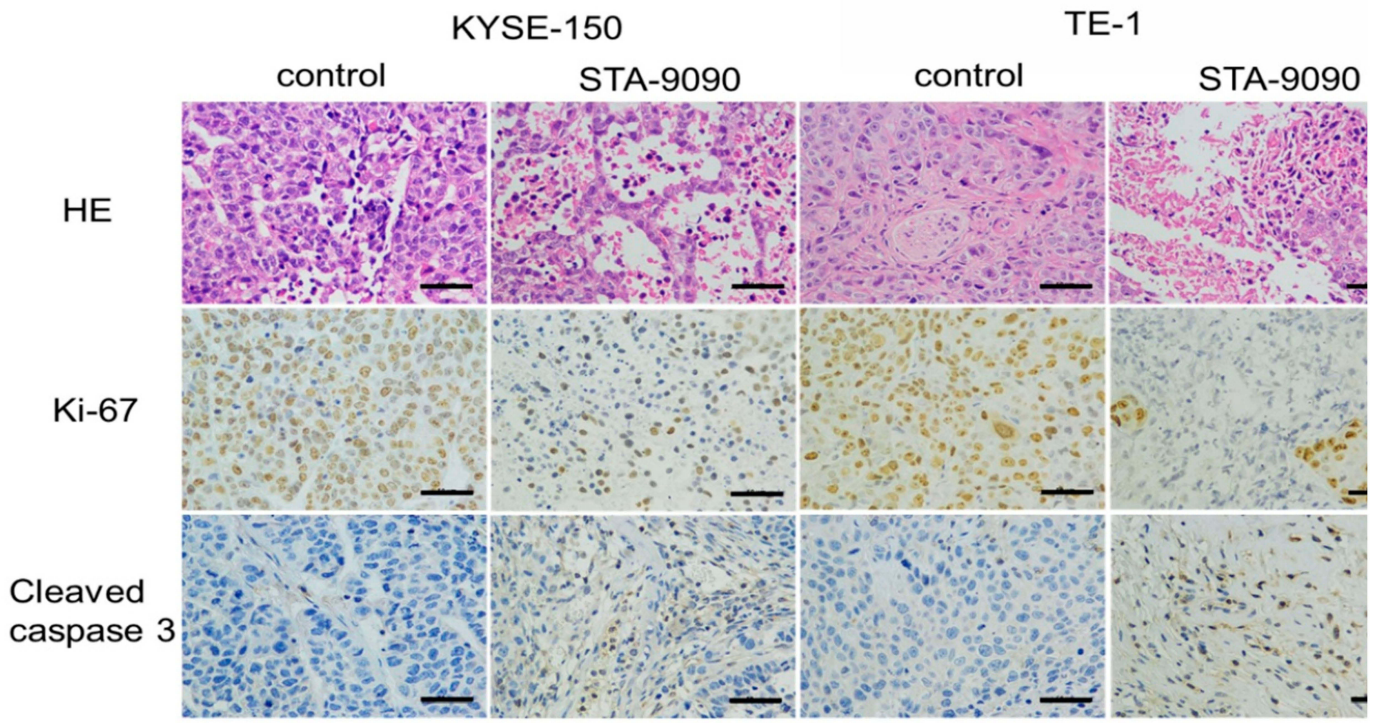

B

TE-1

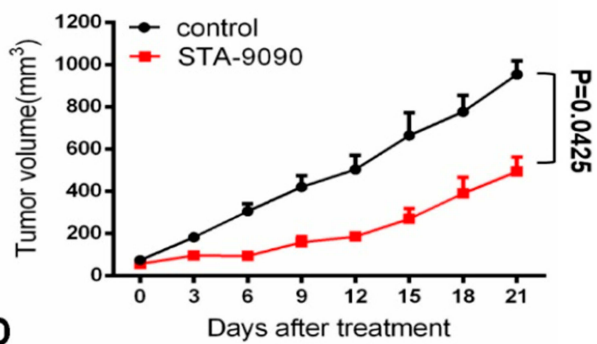

TE-1

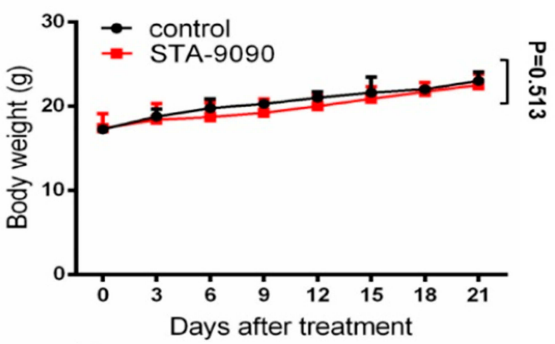

F

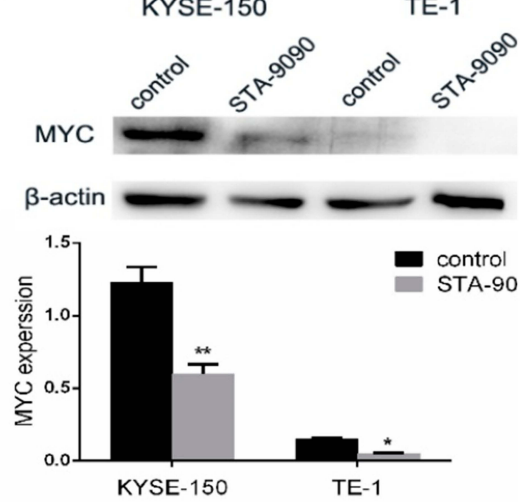

TE-1 
tumors, the body weight was reduced slightly $(\mathrm{p}=0.042)$ in the STA-9090 treatment group comparison with vehicletreated mice (Figure 5C). However, we did not discover any morbidity or decreased activity in mice. There was no significant difference in body weight between two groups of mice with TE-1 tumors $(\mathrm{p}=0.513$ ) (Figure 5D). H\&E staining of tumor sections showed that STA-9090 treatment decreased tumor cell density. In addition, the IHC assay revealed that STA-9090 treatment significantly inhibited the expression of $\mathrm{Ki}-67$ and increased apoptosis (cleaved Caspase 3 staining) in tumors (Figure 5G). STA9090 treatment led to reduced expression of MYC in both KYSE-150 and TE-1 CDXs' tumors (Figure 5F).

\section{STA-9090 Inhibits Tumor Growth in ESCC Patient-Derived Xenograft (PDX) Models}

We established a preclinical PDX model based on samples from ESCC to further assess the antitumor potential of STA9090 treatment in vivo (Figure 6A). As shown in Figure 6B, RNA-sequencing of the samples revealed that the transcriptome of PDX models recapitulated that of the parental tumor tissue $(\mathrm{P}<0.001)$. We also confirmed high MYC expression in passaged tumors in vivo (Figure 6C). Treatments were initiated when tumor volume reached $5 \mathrm{~mm}$ and STA-9090 strongly inhibited tumor growth (Figure 6D). The IHC results revealed that STA-9090 treatment significantly inhibited the expression of $\mathrm{Ki}-67$ and increased apoptosis (cleaved Caspase 3 staining, Figure 6E). The expression of MYC was also decreased following STA-9090 treatment (Figure 6F and G). In order to determine the sensitivity of high MYC expression to STA-9090 treatment, we established PDX models with MYC-low expression ESCC tumors and treated them with STA-9090, we noted that STA-9090 treatment did not have any beneficial effect (Supplementary Figure 4). These data were consistent with the concept that high MYC expressing tumors were more responsive to STA-9090 treatment.

\section{Discussion}

Currently, there is no effective targeted drugs for advanced ESCC. In this study, we demonstrated that the HSP90 inhibitor, STA-9090, possesses remarkable inhibitory effects on the proliferation/growth of ESCC through in vitro and in vivo experiments. The strong antitumor effect of STA-9090 exhibited in ESCC highly expressing MYC was achieved by targeting the HSP90-MYC axis. Fundamental evidence of high MYC expression in ESCC cancer tissues and its interaction with HSP90 was also presented in this study to form the underlying mechanism of STA-9090 as a potential anti-ESCC agent.

Our analysis of ESCC TMA $(n=107)$ and ESCC cancer tissues found that MYC was overexpressed and positively associated with HSP90 expression in ESCC tissues. Furthermore, we also showed that HSP90 function inhibited with the pharmacologic HSP90 inhibitor-STA-9090 was able to directly downregulate MYC leading to a change in MYC transcription and destabilization, further resulting in a significant growth inhibition in MYC-overexpressed ESCC cells. These data suggested that targeting the HSP90MYC axis to destabilize MYC could be a novel therapeutic strategy for ESCC.

Currently, surgery, chemotherapy and radiotherapy, are the mainstream treatments for ESCC and there are no specific targeted drugs for treatment of ESCC. ${ }^{6}$ MYC has been reported to be overexpressed in 50-60\% of all ESCC patients, and suppression of MYC was shown to reverse tumorigenesis in retinoblastoma, breast cancer, hepatocellular, pancreatic cancer and cervical cancer. $^{26-33}$ However, targeting MYC per se has proven to be very challenging because of its intrinsically disordered nature. Therefore, many researchers have looked at inhibiting MYC indirectly by blocking MYC transcription, blocking mRNA translation or targeting regulators of MYC protein stability. ${ }^{34}$ Here we report that compared to adjacent normal tissues, MYC was overexpressed in ESCC cancer tissues, as analyzed by Western blot and immunohistochemistry staining. Similarly, MYC was highly expressed in ESCC KYSE-150 and Eca-109 cell lines.

MYC was first reported as a client protein of HSP90 in mouse fibroblasts. ${ }^{35}$ The HSP90-MYC interaction has been shown in many cancers, including but not limited to, breast cancer, mantle cell lymphoma and B-cell lymphomas. ${ }^{36}$ In our study, we showed that ESCC treatment efficacy may be improved by targeting the HSP90-MYC axis. Specifically, in ESCC cells, we inhibited HSP90 function with the pharmacological inhibitor-STA-9090, leading to a decrease in MYC and reduction of cell reproductive capacity. Compared to the first generation of HSP90 inhibitors which demonstrated unacceptable adverse effects, STA-9090 treatment has reduced risk of heart, eyes, and liver toxicities with multiple ongoing clinical trials for different cancer types. ${ }^{37,38}$ As reported, STA9090 inhibited cell growth by inducing cell cycle arrest in some cancers, including thyroid cancer, gastric cancer and ovarian cancer. ${ }^{39-41}$ Here, we showed that STA-9090 strongly inhibited growth in MYC-overexpressed ESCC cells. However, STA-9090 did not benefit TE-1 cells with low 
A

ESCC patients

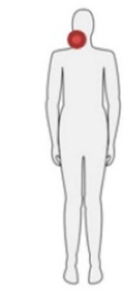

$\mathrm{P} 1, \mathrm{P} 2, \mathrm{P} 3 \ldots \ldots$

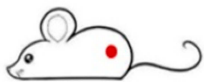

surgery Transplant
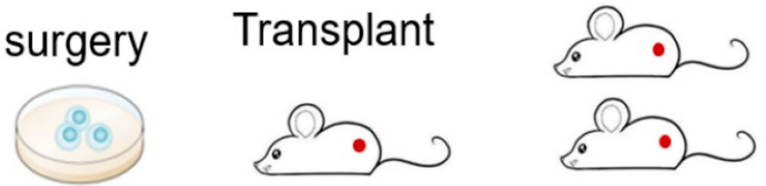

Treatment

21 days

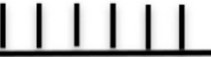

STA-9090

(25mg/kg)
B

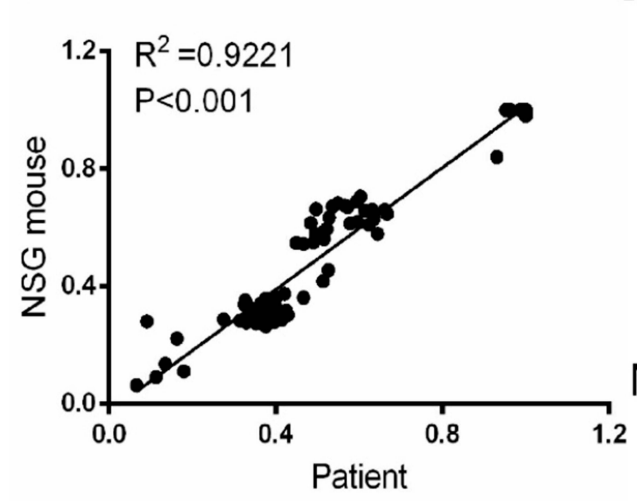

D

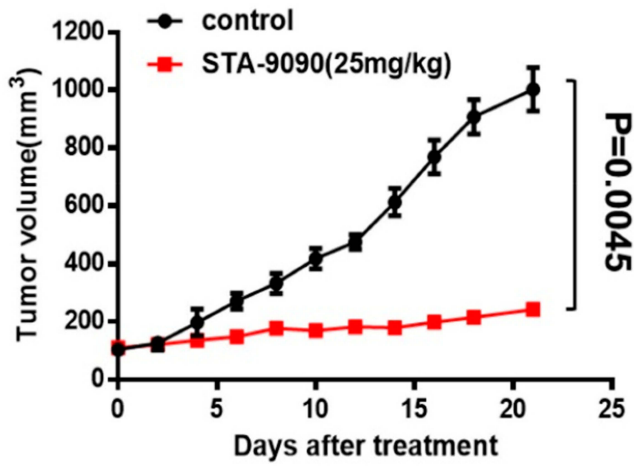

F

G

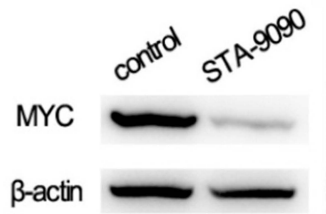

C
$\mathrm{HE}$

P1

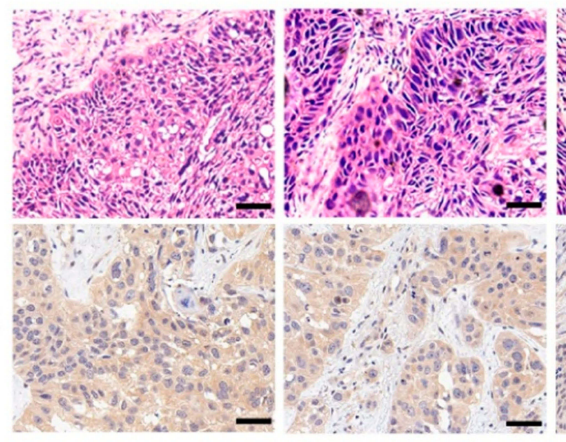

E

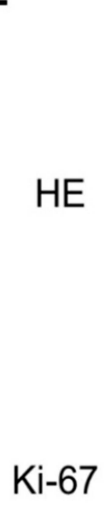

Treatment start
(tumor $\varnothing 5 \mathrm{~mm}$ )

P3

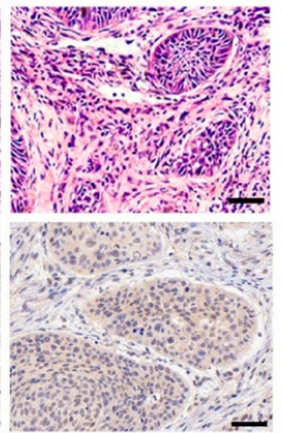

STA-9090

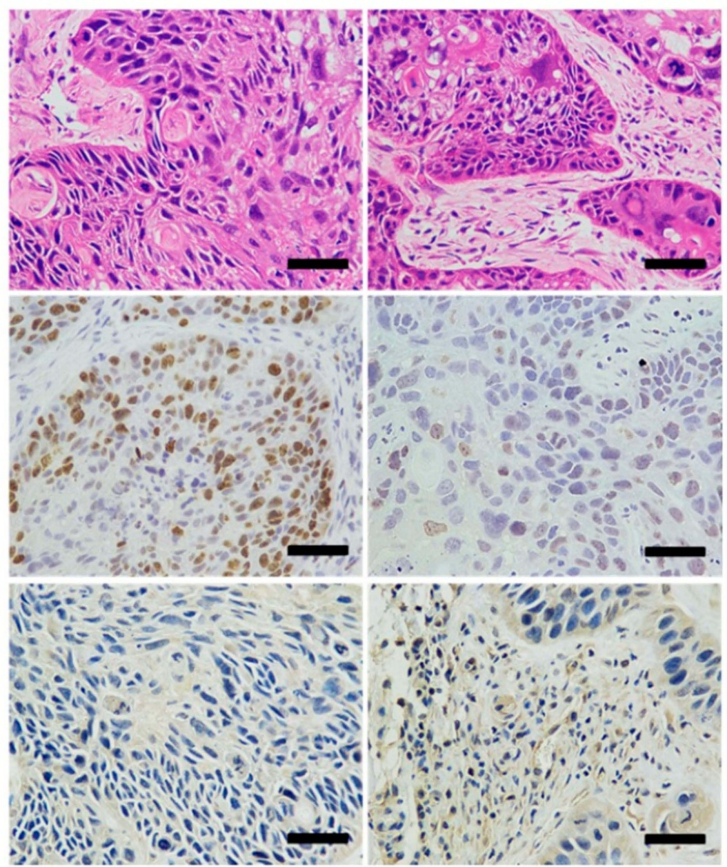

Figure 6 The effects of STA-9090 treatment on ESCC PDX pre-clinical animal models. (A) STA-9090 treatment protocol for ESCC PDX models. Red arrow: start of the treatment. (B) Total RNA was extracted from the patient samples and the matching PDXs. (C) ESCC PDX models retained MYC expression during in vivo passaging of PIP3 generations. Scale bars represent $50 \mu \mathrm{m}$ (D) Effect of STA-9090 treatment on tumor growth in ESCC PDX mice. (E) Histologic analysis of tumors, including H\&E staining, immunohistochemical detection of Ki67 or cleaved Caspase 3. Scale bars represent $50 \mu \mathrm{m}$. (F, G) The protein level of MYC after STA-9090 treatment. $* * * * P<0.000$ I. 
MYC expression showing no cell-proliferation inhibition in vitro. These findings strongly supported that MYCoverexpressed cells are more sensitive to STA-9090 treatment. Importantly, cell cycle analysis revealed that STA-9090 induces G0/G1 phase arrest in ESCC cells. The fact that, in our experiments, inhibition of ESCC cell proliferation after treatment with STA-9090 was achieved through G0/G1 cell cycle arrest, is consistent with previously reported results in melanoma. ${ }^{42}$ We also found that in addition to cell cycle arrest, STA-9090 induced significant apoptosis in MYCoverexpressed ESCC cells, including increased BAX expression and reduced BCL-2 expression. STA-9090 influenced multiple key regulators of cell cycle progression and apoptosis. We also tested the growth of ESCC cells with MYCsilence expression, and we showed cell proliferation was significantly decreased.

An important finding of our study was that STA-9090 showed very strong in vivo antitumor activity against ESCC in both ESCC xenograft mice and human ESCC PDX models $(\mathrm{p}<0.01, \mathrm{p}=0.0017$ in KYSE-150 induced tumors, and $\mathrm{p}=0.0045$ in PDX model), indicating its potential therapeutic application in the treatment of ESCC. Further, we found that the in vivo antitumor effect of STA-9090 exhibited in STA-9090 insensitive ESCC cell line TE-1 tumor. As shown in Figure $5 \mathrm{~B}$ and $\mathrm{E}$, although the magnitude of inhibition was weaker, STA-9090 treatment led to significant in vivo inhibition of TE-1 tumor growth $(\mathrm{p}=0.0425)$. This is consistent with previous findings from the studies by Lin et al who observed that STA-9090 suppressed tumor growth of both sensitive (8505C) and insensitive (TT) human thyroid cancer in vivo. ${ }^{41}$ This may be explained by the determination of a cell line sensitivity to STA-9090 is a relative measure. The advantage using this PDX model type in this study, is that pharmacology studies conducted with PDX models are more predictive for clinical outcome compared to conventional xenograft mouse models. ${ }^{43}$ Fresh tissues from patients with ESCC who had not undergo any preoperative chemoradiotherapy were transplanted subcutaneously into NSG mice. When we further examined the MYC expression in ESCC xenograft mice and PDX models based on the therapeutic outcome, we found a clear decrease in MYC expression in the tumor tissues, suggesting that the antitumor action of STA-9090 was achieved through disrupting the HSP90-MYC axis.

Despite advantages mentioned above, there are some limitations in using the CDX models derived from ESCC tissues/cells. This cell-xenograft model does not represent the unique features of each cancer patient i.e., they do not reflect the patient's tumor original structural and heterogeneity. Human ESCC PDX models, were the xenografts

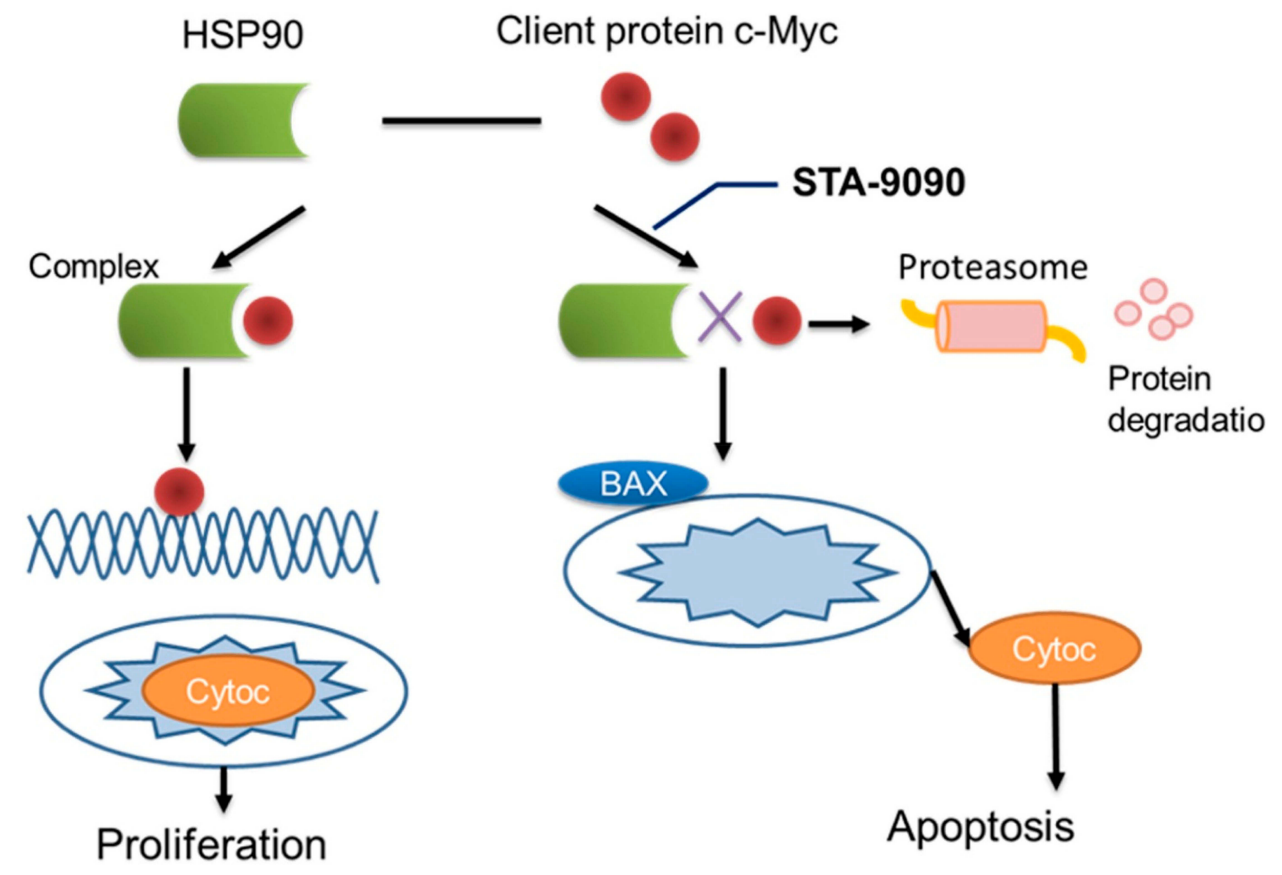

Figure 7 Interactions between MYC and HSP90 and its inhibitor STA-9090. HSP90 binds to client protein MYC involved in ESCC to generate a "complex" that contributes to the stabilization and maturation of those proteins, promoting cellular function. HSP90 inhibitor STA-9090 prevents binding of c-Myc to HSP90, where c-Myc is subsequently degraded by the proteasome, leading to apoptosis. 
are derived from patient tissue, highly preserve the histological features, genomic characters and heterogeneity of patients' tumors. Although the PDX model in our experiments was limited to two patient samples with high expression and low expression of MYC respectively, these results supported our findings using ESCC cell culture xenografts in the PDX mouse model.

\section{Conclusion}

In summary, our study provides data to support that inhibited/reduced expression of MYC in ESCC cells suppresses proliferation and promotes ESCC apoptosis/cell death in vivo and in vitro. Although pharmacologic inhibitors of MYC as therapeutic cancer agents are underdeveloped, MYC as a chaperone of HSP90 in ESCC presents an important opportunity to design rational and targeted protein-protein interactions, like HSP90-MYC, as a promising alternative strategy for MYC-positive ESCC patients (Figure 7). Our study provides fundamental evidence supporting future preclinical and clinical testing of STA-9090 in MYC-positive ESCC. Although the use of other HSP90 inhibitors in cancer patients has not shown impressive clinical activity at present, ${ }^{38,44,45}$ it is possible that STA-9090 is an agent with high antitumor efficacy against MYC overexpressing ESCC, i.e. a subset of ESCC patients who could potentially benefit from this drug.

\section{Abbreviations}

BCA, Bicinchoninic acid; CDX, Cell-derived xenograft; CHX, Cycloheximide; c-Myc, MYC; Co-IP, Coimmunoprecipitation assay; DMEM, Dulbecco Minimal Essential Medium; EC, Esophagus Carcinoma; EAC, Esophagus Adenocarcinoma; ESCC, Esophageal Squamous Cell Carcinoma; FBS, Fetal Bovine Serum; H\&E staining, Hematoxylin and Eosin staining; HEEC, Non-carcinoma Human Esophageal Epithelial cell; HSP90, Heat Shock Protein 90; PBS, Phosphate buffered saline; PDX, patientderived xenograft; PI, Propidium Iodide; qRT-PCR, Quantitative real time-PCR; RPMI-1640, Roswell Park Memorial Institute-1640; RIPA, Radio Immunoprecipitation Assay; siRNA, Short interfering RNA; TMA, tissue microarrays.

\section{Acknowledgments}

The authors would like to thank Dr Eileen McGowan of the University of Technology Sydney for providing critical reading of the manuscript.

\section{Funding}

This work was supported by research grants from the Yuexiu District Science and Technology Program of Guangzhou (Grant numbers: 2017WS002); Special Innovative Projects of Guangdong Province (Grant number: 2017KTSCX104); and Science and Technology Program of Guangzhou City (Grant number: 2018059).

\section{Disclosure}

The authors report no conflicts of interest in this work.

\section{References}

1. Bray F, Ferlay J, Soerjomataram I, Siegel RL, Torre LA, Jemal A. Global cancer statistics 2018: GLOBOCAN estimates of incidence and mortality worldwide for 36 cancers in 185 countries. CA Cancer J Clin. 2018;68(6):394-424. doi:10.3322/caac.v68.6

2. Stewart B, Wild CP. World Cancer Report 2014. Lyon, France: WHO Press; 2017.

3. Smyth EC, Lagergren J, Fitzgerald RC, et al. Oesophageal cancer. Nat Rev Dis Primers. 2017;3(1):17048. doi:10.1038/nrdp.2017.48

4. Francesca I, Doroudchi MM, Ismail N, Carreno A, Griner E, Lim MA. Registered report: interactions between cancer stem cells and their niche govern metastatic colonization. Elife. 2015;4:e06938. doi:10.7554/eLife.06938

5. Zhang L, Ma J, Han Y, et al. Targeted therapy in esophageal cancer. Expert Rev Gastroenterol Hepatol. 2016;10(5):595-604. doi:10.1586/ 17474124.2016.1140036

6. Domper Arnal MJ, Ferrandez Arenas A, Lanas Arbeloa A. Esophageal cancer: risk factors, screening and endoscopic treatment in Western and Eastern countries. World J Gastroenterol. 2015;21 (26):7933-7943. doi:10.3748/wjg.v21.i26.7933

7. Grandori C, Cowley SM, James LP, Eisenman RN. The Myc/Max/ Mad network and the transcriptional control of cell behavior. Annu Rev Cell Dev Biol. 2000;16(1):653-699. doi:10.1146/annurev.cellbio. 16.1.653

8. Pelengaris S, Khan M, Evan G. c-MYC: more than just a matter of life and death. Nat Rev Cancer. 2002;2(10):764-776. doi:10.1038/ nrc904

9. Dang CV, O’Donnell KA, Zeller KI, Nguyen T, Osthus RC, Li F. The c-Myc target gene network. Semin Cancer Biol. 2006;16(4):253-264. doi:10.1016/j.semcancer.2006.07.014

10. Fernandez PC, Frank SR, Wang L, et al. Genomic targets of the human c-Myc protein. Genes Dev. 2003;17(9):1115-1129.

11. Lian Y, Niu X, Cai $\mathrm{H}$, et al. Clinicopathological significance of c-MYC in esophageal squamous cell carcinoma. Tumour Biol. 2017;39(7):1010428317715804. doi:10.1177/1010428317715804

12. Wang J, Liu Z, Wang Z, et al. Targeting c-Myc: JQ1 as a promising option for c-Myc-amplified esophageal squamous cell carcinoma. Cancer Lett. 2018;419:64-74. doi:10.1016/j.canlet.2018.01.051

13. Wiech H, Buchner J, Zimmermann R, Jakob U. Hsp90 chaperones protein folding in vitro. Nature. 1992;358(6382):169-170. doi:10. $1038 / 358169 \mathrm{a} 0$

14. Whitesell L, Lindquist SL. HSP90 and the chaperoning of cancer. Nat Rev Cancer. 2005;5(10):761-772. doi:10.1038/nrc1716

15. Yan H, Li B, Fan T, Jiang S, Wang R, Sun M. Clinical significance of serum dynamics of HSP90a level in esophageal squamous cell carcinoma patients treated with definitive chemoradiotherapy. Cancer Biomark. 2017;19(2):185-192. doi:10.3233/CBM-160502

16. Trepel J, Mollapour M, Giaccone G, Neckers L. Targeting the dynamic HSP90 complex in cancer. Nat Rev Cancer. 2010;10 (8):537-549. doi:10.1038/nrc2887 
17. Taipale M, Jarosz DF, Lindquist S. HSP90 at the hub of protein homeostasis: emerging mechanistic insights. Nat Rev Mol Cell Biol. 2010;11(7):515-528. doi:10.1038/nrm2918

18. Lee J, Zhang LL, Wu W, et al. Activation of MYC, a bona fide client of HSP90, contributes to intrinsic ibrutinib resistance in mantle cell lymphoma. Blood Adv. 2018;2(16):2039-2051. doi:10.1182/ bloodadvances. 2018016048

19. Jhaveri K, Modi S. Ganetespib: research and clinical development. Onco Targets Ther. 2015;8:1849-1858. doi:10.2147/OTT.S65804

20. Proia DA, Foley KP, Korbut T, et al. Multifaceted intervention by the Hsp90 inhibitor ganetespib (STA-9090) in cancer cells with activated JAK/STAT signaling. PLoS One. 2011;6(4):e18552. doi:10.1371/ journal.pone. 0018552

21. Proia DA, Zhang C, Sequeira M, et al. Preclinical activity profile and therapeutic efficacy of the HSP90 inhibitor ganetespib in triple-negative breast cancer. Clin Cancer Res. 2014;20(2):413-424. doi:10.1158/1078-0432.CCR-13-2166

22. Ramalingam S, Goss $\mathrm{G}$, Rosell $\mathrm{R}$, et al. A randomized Phase II study of ganetespib, a heat shock protein 90 inhibitor, in combination with docetaxel in second-line therapy of advanced non-small cell lung cancer (GALAXY-1). Ann Oncol. 2015;26(8):1741-1748. doi:10.1093/annonc/ $\operatorname{mdv} 220$

23. Shimamura T, Perera SA, Foley KP, et al. Ganetespib (STA-9090), a nongeldanamycin HSP90 inhibitor, has potent antitumor activity in in vitro and in vivo models of non-small cell lung cancer. Clin Cancer Res. 2012;18(18):4973-4985. doi:10.1158/1078-0432.CCR-11-2967

24. Garcia-Carbonero R, Carnero A, Paz-Ares L. Inhibition of HSP90 molecular chaperones: moving into the clinic. Lancet Oncol. 2013;14 (9):e358-e369. doi:10.1016/S1470-2045(13)70169-4

25. Kim T, Jeon YJ, Cui R, et al. Role of MYC-regulated long noncoding RNAs in cell cycle regulation and tumorigenesis. J Natl Cancer Inst. 2015;107(4). doi:10.1093/jnci/dju505

26. Goldman JW, Raju RN, Gordon GA, et al. A first in human, safety, pharmacokinetics, and clinical activity Phase I study of once weekly administration of the Hsp90 inhibitor ganetespib (STA-9090) in patients with solid malignancies. BMC Cancer. 2013;13(1):152. doi:10.1186/1471-2407-13-152

27. Yu N, Chen P, Wang Q, et al. Histone deacetylase inhibitors differentially regulate c-Myc expression in retinoblastoma cells. Oncol Lett. 2020;19(1):460-468. doi:10.3892/ol.2019.11111

28. Zhang Z, Liu M, Hu Q, et al. FGFBP1, a downstream target of the FBW7/c-Myc axis, promotes cell proliferation and migration in pancreatic cancer. Am J Cancer Res. 2019;9(12):2650-2664.

29. Felsher DW, Bishop JM. Reversible tumorigenesis by MYC in hematopoietic lineages. Mol Cell. 1999;4(2):199-207. doi:10.1016/S10972765(00)80367-6

30. Jain M, Arvanitis C, Chu K, et al. Sustained loss of a neoplastic phenotype by brief inactivation of MYC. Science. 2002;297 (5578):102-104. doi:10.1126/science.1071489

31. Pelengaris S, Khan M, Evan GI. Suppression of Myc-induced apoptosis in beta cells exposes multiple oncogenic properties of Myc and triggers carcinogenic progression. Cell. 2002;109(3):321-334. doi:10.1016/S0092-8674(02)00738-9
32. Marinkovic D, Marinkovic T, Mahr B, Hess J, Wirth T. Reversible lymphomagenesis in conditionally c-MYC expressing mice. Int $J$ Cancer. 2004;110(3):336-342. doi:10.1002/(ISSN)1097-0215

33. Zhang L, Fu Y, Guo H. c-Myc-induced long non-coding RNA small nucleolar RNA host gene 7 regulates glycolysis in breast cancer. J Breast Cancer. 2019;22(4):533-547. doi:10.4048/jbc.2019.22.e54

34. Whitfield JR, Beaulieu ME, Soucek L. Strategies to inhibit Myc and their clinical applicability. Front Cell Dev Biol. 2017;5:10. doi:10.3389/fcell.2017.00010

35. Carystions GD, Kandouz M, Alaoui-Jamali MA, Batist G. Unexpected induction of the human connexin 43 promoter by the ras signaling pathway is mediated by a novel putative promoter sequence. Mol Pharmacol. 2003;63(4):821-831. doi:10.1124/mol.63.4.821

36. Lee YC, Chang WW, Chen YY, et al. Hsp90alpha mediates BMI1 expression in breast cancer stem/progenitor cells through facilitating nuclear translocation of c-Myc and EZH2. Int J Mol Sci. 2017;18(9). doi:10.3390/ijms18091986

37. Subramaniam DS, Liu SV, Crawford J, et al. A phase Ib/II study of ganetespib with doxorubicin in advanced solid tumors including relapsed-refractory small cell lung cancer. Front Oncol. 2018;8:64. doi:10.3389/fonc.2018.00064

38. Shah S, Luke JJ, Jacene HA, et al. Results from phase II trial of HSP90 inhibitor, STA-9090 (ganetespib), in metastatic uveal melanoma. Melanoma Res. 2018;28(6):605-610. doi:10.1097/ CMR.0000000000000509

39. Lee H, Saini N, Parris AB, Zhao M, Yang X. Ganetespib induces G2/ $\mathrm{M}$ cell cycle arrest and apoptosis in gastric cancer cells through targeting of receptor tyrosine kinase signaling. Int J Oncol. 2017;51 (3):967-974. doi:10.3892/ijo.2017.4073

40. Gabbasov R, Benrubi ID, O'Brien SW, et al. Targeted blockade of HSP90 impairs DNA-damage response proteins and increases the sensitivity of ovarian carcinoma cells to PARP inhibition. Cancer Biol Ther. 2019;20(7):1035-1045. doi:10.1080/15384047.2019.159 5279

41. Lin SF, Lin JD, Hsueh C, et al. Efficacy of an HSP90 inhibitor, ganetespib, in preclinical thyroid cancer models. Oncotarget. 2017;8(25):41294-41304. doi:10.18632/oncotarget.17180

42. Wu X, Marmarelis ME, Hodi FS, Smalley K. Activity of the heat shock protein 90 inhibitor ganetespib in melanoma. PLoS One. 2013;8(2):e56134. doi:10.1371/journal.pone.0056134

43. Cho SY, Kang W, Han JY, et al. An integrative approach to precision cancer medicine using patient-derived xenografts. Mol Cells. 2016;39 (2):77-86.

44. Goyal L, Wadlow RC, Blaszkowsky LS, et al. A phase I and pharmacokinetic study of ganetespib (STA-9090) in advanced hepatocellular carcinoma. Invest New Drugs. 2015;33(1):128-137. doi:10.1007/s10637-014-0164-8

45. Goyal L, Chaudhary SP, Kwak EL, et al. A Phase 2 clinical trial of the heat shock protein 90 (HSP 90) inhibitor ganetespib in patients with refractory advanced esophagogastric cancer. Invest New Drugs. 2020. doi:10.1007/s10637-019-00889-y

\section{Publish your work in this journal}

OncoTargets and Therapy is an international, peer-reviewed, open access journal focusing on the pathological basis of all cancers, potential targets for therapy and treatment protocols employed to improve the management of cancer patients. The journal also focuses on the impact of management programs and new therapeutic

Submit your manuscript here: https://www.dovepress.com/oncotargets-and-therapy-journa agents and protocols on patient perspectives such as quality of life, adherence and satisfaction. The manuscript management system is completely online and includes a very quick and fair peer-review system, which is all easy to use. Visit http://www.dovepress.com/ testimonials.php to read real quotes from published authors. 